\title{
La innovación tecnológica producto del I+D+I como estrategia para el desarrollo de empresas familiares de la industria carrocera de Tungurahua
}

\section{The technological innovation product of the $R+D+I$ as strategy for the development of family enterprises of the carrocera industry of Tungurahua}

\author{
Eufemia Alejandrina Ramos Viteri ${ }^{1 *}$ \\ ${ }^{1}$ Universidad Técnica de Ambato, Ecuador \\ *eufemiaaramos@uta.edu.ec \\ DOI: https://doi.org/10.26871/killkana_social.v4i1.530
}

\begin{abstract}
Resumen
Una innovación tecnológica surge tras la utilización de la tecnología como medio para introducir un cambio en la empresa, tanto a productos como a procesos. En tal virtud, el objetivo del presente estudio se centra en determinar la vinculación entre la innovación tecnológica y el desarrollo de empresas familiares del sector carrocero. Para ello, la metodología empleada esta dada bajo un enfoque cuantitativo, la misma que permitió a través de la recolección de información conseguir los datos históricos en cuanto se refiere a los ingresos y costos de cada una de las empresas en estudio (ALME, MAMBUS, IMPA, IMPEDEPSA, PILLAPA). Asimismo, se realizó una investigación bibliográfica con la finalidad de sustentar de forma teórica cada una de las variables inmersas en el estudio (innovación tecnológica y desarrollo empresarial). En relación al alcance, éste fue de carácter exploratorio dado que no existen estudios similares en el sector carrocero de la provincia. Con estos antecedentes, entre los principales resultados encontrados se pudo determinar que durante los últimos años cada una de las empresas evaluadas destinaron recursos para la adquisición de nuevas tecnologías específicas para cada tipo de bus (bus interprovincial, bus tipo y bus escolar) con lo cual se registró una considerable disminución de los costos totales por empresa lo cual permitió estimar los beneficios a nivel de sector para los diferentes años.
\end{abstract}

Palabras clave: Costos, desarrollo empresarial, ingresos, innovación tecnológica.

\begin{abstract}
A technological innovation arises after the use of technology as a means to introduce a change in the company, both to products and processes. Accordingly, the objective of this study is to determine the link between technological innovation and the development of family businesses in the car industry. For this, the methodology used is given under a quantitative approach, the same that allowed through the collection of information to obtain historical data as regards the income and costs of each of the companies under study (ALME, MAMBUS, IMPA, IMPEDEPSA, PILLAPA). Likewise, a bibliographical investigation was carried out with the purpose of sustaining theoretically each one of the variables immersed in the study (technological innovation and business development). In relation to the scope, it was exploratory in nature since there are no similar studies in the province's body sector. With this background, among the main results found, it was determined that during the last years each of the companies evaluated allocated resources for the acquisition of new specific technologies for each type of bus (interprovincial bus, type bus and school bus) with which There was a considerable decrease in total costs per company which allowed estimating the benefits at the sector level for the different years.
\end{abstract}

Keywords: Business development, costs, income, technological innovation.

\section{Introducción}

La innovación se considera uno de los factores más importantes para el éxito en el entorno competitivo actual y, como resultado, las empresas buscan continuamente mode- los de innovación efectivos para fortalecer su ventaja competitiva, así como, aumentar el rendimiento (Robayo Acuña, 2016). La innovación y el desempeño tienen una etapa central en la literatura sobre emprendimiento estratégico, donde la innovación tecnológica, en particular, se considera 
como un agente impulsor fundamental para fomentar la ventaja competitiva de las empresas u organizaciones. En este contexto, la tecnología se puede definir como un tipo de conocimiento aplicado al desarrollo y comercialización de nuevos productos o servicios (Vélez Romero y Ortiz Restrepo, 2016).

Las decisiones estratégicas relacionadas con el lado de la tecnología se refieren a varios aspectos, tales como: la intensidad y el tiempo con el que los nuevos productos o servicios deben comercializarse en el mercado; si la empresa debe dedicar recursos al desarrollo de productos o servicios nuevos radical o incrementalmente; si el desarrollo de nuevos productos está dirigido más por el conocimiento tecnológico que por el conocimiento del mercado; si los procesos de innovación deben implicar o no compartir el conocimiento a través de redes con otras organizaciones.

La innovación ha sido considerada fundamental por mucho tiempo para el crecimiento empresarial, especialmente en el contexto de la globalización y la hipercompetencia. Las empresas familiares representan una parte significativa del total de las empresas en todo el mundo, estimadas entre el 65 y el $90 \%$. En este sentido, este tipo de empresas se encuentran obligadas a enfrentar una mayor vulnerabilidad como consecuencia de un complejo entrelazamiento entre los objetivos empresariales y familiares. Esta relación desafiante plantea importantes preguntas de investigación en términos de estructura organizacional, estilos de gestión y desempeño e innovación (Cadena, Pereira, y Pérez, 2019).

Las organizaciones que destinan mayores recursos en la denominada I+D suelen ser aquellas que innovan y generan nuevo conocimiento en la rama o sector en la que se desenvuelven. En tal virtud, para poder innovar, una organización debe tener a su disposición no únicamente recursos de tipo monetario sino también recursos humanos que le permitan en el corto tiempo generar nuevos conocimientos que aporten a la gestión empresarial. Resulta común hoy en día ver cómo empresas que se encuentran dentro de un alto nivel tienen a su disposición trabajadores con un alto grado de formación, instrucción y capacitación, que a través de su gestión han permitido consolidar a las empresas en líderes en el mercado (Cea, 2016).

Adicionalmente, se puede argumentar que la investigación y desarrollo (I+D) se ve reflejado a través de la innovación empresarial, que constituye un factor de gran relevancia para el desempeño de cualquier tipo de empresa. Para el caso de estudio, que constituye el sector carrocero, Ecuador cuenta con un número interesante de organizaciones pertenecientes a esta industria, sin embargo la provincia que tiene mayor destaque es Tungurahua dado que el $65 \%$ de la producción nacional se encuentra en ella, por lo que hoy en día se presenta como un sector clave dentro de la dinámica económica no sólo por la entrada de recursos y el empleo que genera, sino también por la mejora que ha generado a nivel de bienestar, industrialización, desarrollo y urbanización de la región (Mantilla, Vilcacundo, Ruiz, y Mayorga, 2016).

Bajo este contexto, la finalidad del presente estudio es determinar la vinculación entre la innovación tecnológica y el desarrollo de empresas familiares del sector carrocero utilizando los estados financieros con la finalidad de determinar un punto de equilibrio entre ingresos y costos totales que han sido generados en los periodos de estudio. De esta manera, relacionar los beneficios obtenidos y contrastar si existen diferencias significativas al insertar aspectos tecnológicos, es decir innovación tecnológica en cada uno de los productos que son ofertados al mercado local y nacional.

\subsection{Innovación tecnológica}

La innovación tecnológica y la implementación apropiada de nuevas tecnologías son una parte fundamental del proceso de desarrollo de todas las naciones. En tal virtud, la innovación tecnológica se puede considerar como un proceso que presenta su fundamento en la ciencia, la tecnología y el sistema, en este sentido, este proceso incluye directamente varios elementos que afectan y se ven afectados por las capacidades internas de la empresa, su trabajo y su capacidad de aprendizaje e influenciados por sus factores externos como los ambientales (Da Silva Tavares y Schneck de Paula Pessoa, 2014).

En el contexto actual de la globalización económica por el cual atraviesa el mundo, resalta la importancia que tienen las actividades de investigación y desarrollo tecnológico (I+D) en el posicionamiento competitivo de las empresas (Bonilla Jurado, Oña Sinchiguano, y López Núñez, 2018). En este sentido, la tecnología constituye una de las principales fuentes de competitividad e integra aspectos tales como experiencia, conocimiento, equipo,instalaciones, software, entre otros que permite la generación de nuevos productos, procesos, servicios y sistemas, así como importantes mejoras a los que ya existen. En tal virtud, el desarrollo tecnológico, sin importar su fuente, está determinado por necesidades sociales o demandas del mercado y tiene una fuerte implicación económica para cada una de las empresas u organizaciones (De Massis, Frattini, y Lichtenthaler, 2012).

En este contexto, la innovación tecnológica supone para la organización la introducción de un cambio técnico ya sea en los productos o en sus procesos, este criterio coincide con lo expuesto en el Manual de Oslo donde se asevera que las innovaciones tecnológicas comprenden nuevos productos y nuevos procesos y con ello cambios tecnológicos significativos en ambos casos. No obstante, bajo el criterio de Perozo y Villalobos (2014) la innovación tecnológica, no únicamente tiene que ver con nuevos procesos o productos, sino también con la introducción de nuevas plantas de producción, nueva maquinaria para sus procesos, así como la mercantilización de nuevos productos.

Finalmente, en torno a este tema, la innovación tecnológica es considerado como el proceso encaminado para tratar y resolver problemas centrados principalmente en el 
Tabla 1: El papel de la innovación tecnológica en la gestión empresarial

\begin{tabular}{ll}
\hline & $\begin{array}{l}\text { La aplicación de la innovación tecnológica puede proponer métodos de gestión más diversificados para la gestión } \\
\text { empresarial sobre la base tradicional. Estos métodos de gestión son más simples y convenientes, y pueden hacer que } \\
\text { la gestión interna de la empresa funcione de manera sistemática. Al mismo tiempo, el uso de la innovación tecnológica } \\
\text { también puede llevar a cabo una gestión más ordenada. Por un lado, puede mejorar la eficiencia de la gestión de la seguridad, } \\
\text { la gestión de los empleados y la gestión de archivos, por otro lado, también puede mejorar el nivel de gestión de las } \\
\text { empresas. }\end{array}$ \\
$\begin{array}{l}\text { Mejora la eficiencia de de las empresas } \\
\text { gestión de la }\end{array}$ & $\begin{array}{l}\text { Las empresas que utilizan la innovación tecnológica para la gestión empresarial pueden fortalecer su extensión comercial } \\
\text { en el mercado. Además, se invierten más recursos humanos y financieros en el negocio para crear más oportunidades para } \\
\text { la gestión del negocio y mejorar la eficiencia del negocio. }\end{array}$ \\
\hline $\begin{array}{l}\text { Mejora la eficiencia ope- } \\
\text { rativa de las empresas }\end{array}$ & $\begin{array}{l}\text { La economía de mercado cambia constantemente. En este contexto, las empresas encontrarán algunos problemas nuevos } \\
\text { durante su desarrollo, que tendrán un impacto en el desarrollo futuro. Para resolver estos problemas de manera rápida y } \\
\text { eficiente y lograr un desarrollo a largo plazo, las empresas deben adaptarse a los cambios de la sociedad y la economía de } \\
\text { mercado, y llevar a cabo la innovación tecnológica para apoyar la gestión empresarial de las empresas. }\end{array}$ \\
\hline $\begin{array}{l}\text { Propicia el desarrollo plazo de las empre- } \\
\text { sas }\end{array}$ &
\end{tabular}

Fuente: Adaptado de Haixing (2018)

mercado y sus diversos agentes, implica el intercambio de conocimientos tácito y explícito que facilita el aprendizaje desde distintos escenarios (Herrera González y Gutiérrez Gutiérrez, 2015). Por lo tanto, la adecuada gestión de la tecnología representa una de las claves del éxito en los negocios en la actualidad pues mucho depende de ello para que la organización logre y mantenga una ventaja tecnológica que le permita ser competitiva.

\subsection{Importancia de la tecnología en la estrategia de inno- vación}

De acuerdo con diferentes estudios se busca categorizar las posibilidades estratégicas de la organización en innovación, teniendo como punto de partida el análisis de la jerarquía en la que de acuerdo a las capacidades existentes puede enfocarse dicha estrategia y de la competencia que se registra en el mercado. En este sentido, este análisis parte de la mezcla de tres factores importantes como Proceso - Producto - Mercado. A partir de lo expuesto, Londoño (2015) propone la siguiente clasificación en relación a las estrategias de innovación (ver figura 1).

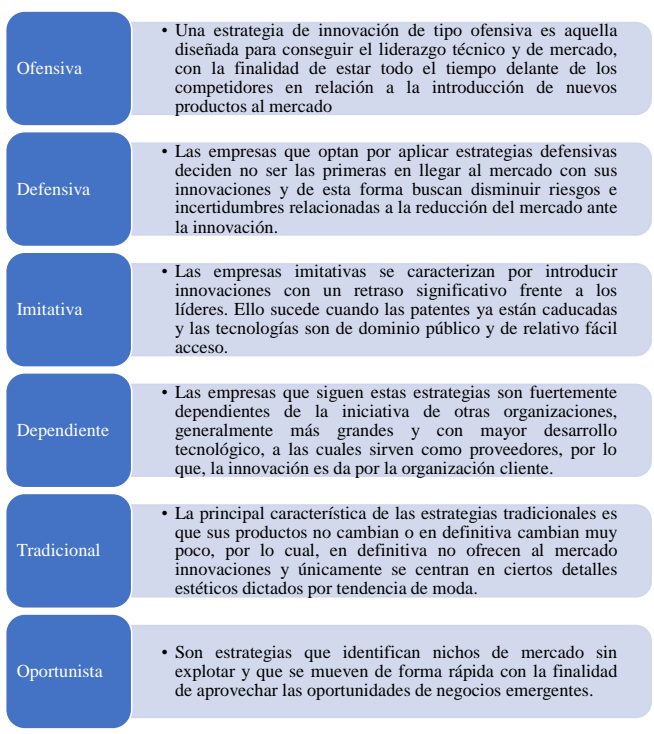

Figura 1: Estrategias de innovación

Fuente:Adaptado de Londoño (2015)
Bajo este contexto, la clasificación expuesta ayuda a identificar distintas opciones que tiene una empresa al establecer sus estrategias de innovación de una forma deliberada, en la cual se supone que todas las variables de mercados, competencia, capacidades empresariales, incluyendo la ciencia y la tecnología se encuentran plenamente definidas (Robledo Velásquez, 2017). Sin embargo, resulta importante recalcar que esta condición de las variables no puede ser adaptable en países con economías emergentes. En este sentido en la figura 1, se clasifican las estrategias de innovación de acuerdo al nivel de desarrollo tecnológico de la organización y el nivel de participación en el mercado, lo que ayuda a clarificar a la categorización de las posibilidades estratégicas de innovación que posee una organización dentro del contexto competitivo donde se desenvuelve (Seclén, 2016).

\subsection{Empresas familiares}

Las empresas controladas por familias son la forma dominante de empresa en todo el mundo, dado que realizan importantes contribuciones al desarrollo socioeconómico y al emprendimiento en la mayoría de los países, sin embargo, a pesar de ello no existe un consenso sólido teniendo en cuenta la definición de empresas familiares (Molina Parra, Botero Botero, y Montoya Monsalve, 2016). A partir de ello, Rahman, Sanguino y Barroso (2017) establecen que la mayoría de las definiciones enmarcan o toman en consideración al menos tres de las siguientes dimensiones: 1) una o varias familias captan una parte importante del capital de la empresa; 2) los miembros de la familia tienen el mayor control sobre la empresa; y 3) los miembros de la familia captan los puestos de alta dirección.

En este sentido, el propósito de las empresas familiares puede enfocarse más hacia la existencia a largo plazo del negocio y retenerlos en manos de la familia, a diferencia de aquellas empresas no familiares quienes tienen propósitos a corto plazo y con mayor orientación hacia la obtención de réditos económicos (Carrasco Hernández y Sánchez Marín, 2014). Este escenario hace que por su condición misma la rentabilidad y el crecimiento de la facturación sean menores, además de presentar menos probabilidades de tener riesgos o de ampliar su oferta. 
Bajo este contexto, la empresa familiar constituye una organización social de gran complejidad, donde conviven la familia y la empresa, entes que se desempeñan como dos subsistemas interdependientes, superpuestos y generadores en determinados momentos de conflictos. En tal virtud, su importancia radica en la realidad socioeconómica de cualquier nación, dado que representan en un agente activo en la regeneración de la estructura económica regional. De esta manera, por un lado, se habla del gran impacto de las empresas familiares en la generación de empleo y riqueza, y por otro, se tratan sus fortalezas y debilidades. En relación a las fortalezas, por ejemplo, se destaca el incremento de la dedicación y compromiso, la identidad de los valores familiares, la transmisión en el tiempo de valores y conocimientos, una mayor reinversión de los beneficios y con ello mayor responsabilidad social; mientras que en relación a las debilidades éstas se ven reflejadas en el riesgo de confusión entre cultura e intereses familiares, conservadurismo y rigidez, autofinanciación y sucesión mal preparada (Quejada Pérez y Ávila Gutiérrez, 2016).

\subsection{Desarrollo empresarial familiar}

El desarrollo empresarial articula distintos factores como el liderazgo, la cultura empresarial, la gestión del conocimiento o innovación con los que el empresario en determinado momento puede llevar a una empresa hacia la consecución de sus objetivos planeados. En este sentido, representa un concepto integrador con el que se puede conseguir un impacto positivo en las empresas a través del reconocimiento de las capacidades del capital humano involucrado. De esta manera, alcanzar un desarrollo empresarial le permitirá al empresario de una empresa familiar aprovechar las oportunidades que se le presentan a la empresa en un entorno globalizado y con alto nivel de competencia (Delfín Pozos y Acosta Márquez, 2016).

Para conseguir este crecimiento, la organización esta en la obligación de desarrollar diferentes estrategias. En este escenario, la tipología más conocida de estrategias de crecimiento corresponde a la estrategia de expansión y la estrategia de diversificación. Donde según Mariz y Calvo (2015) la primera se enfoca en el desarrollo de los productos o mercados tradicionales, o en ambos a la vez, mientras que la diversificación hace referencia a la entrada en nuevos mercados con productos novedosos, suponiendo una cierta ruptura con la situación actual y cambios en los sistemas internos de dirección.

Por otro lado, cuando se trata de una empresa familiar, garantizar la supervivencia y crecimiento del negocio no resulta ser una tarea fácil, no obstante, puede tener una enorme importancia para asegurar la continuidad de la organización en manos de la siguiente generación. Al respecto, Fuentes, Hernández y Vallejo (2008) establecen que dentro de los objetivos de este tipo de estructuras empresariales debería necesariamente considerarse la importancia del crecimiento del negocio con la finalidad de garantizar su continuidad en manos de las futuras generaciones dentro del núcleo familiar. Dado que, se supone que a medida que la familia crece, la empresa también debería crecer y la forma de conseguirlo es a través del diseño y la implementación de estrategias orientadas al crecimiento, en este caso la mejor forma de hacerlo es a través de la innovación tecnológica que demandan los productos actuales, pues de no ser así, el patrimonio empresarial a dejar cada vez experimentaría una reducción considerable, en términos relativos, por lo que, esta estructura organizacional correría el riego de cerrar sus actividades por falta de liquidez (Sandu, 2019).

\section{Materiales y Métodos}

El enfoque de investigación dentro del presente estudio fue de carácter cuantitativo, dado que según Hernández y Mendoza (2018) este estudio se fundamenta en la recolección de datos numéricos derivados de cifras o cantidades implícitas en las variables de estudio, a través de la utilización de operaciones estandarizadas y admitidos por la colectividad científica. Bajo este contexto, a través de la recolección de información se pudo conseguir los datos históricos en cuanto se refiere a los ingresos y costos de cada una de las empresas en estudio, los mismos que fueron sometidos a un análisis estadístico donde se pudo comprobar que la innovación tecnológica si influye en el crecimiento empresarial.

Por otro lado, en relación a la modalidad de investigación fue necesario desarrollar una investigación bibliográfica, pues según Gómez, Fernando, Aponte y Betancourt (2014) mencionan que este apartado constituye una etapa indispensable de todo proyecto de investigación y debe garantizar la obtención de la información más importante en el campo de estudio, de un universo de documentos que puede ser muy extenso. En este sentido, se procesó la información recabada en diferentes fuentes de información secundarias; es decir, libros, revistas, investigaciones realizadas con anterioridad, entre otros documentos públicos y privados, los mismos que fueron procesados en su totalidad escogiendo información relevante que se encuentra descrita a lo largo del presente manuscrito.

Asimismo, en cuanto al alcance del estudio, éste se centra en un nivel exploratorio; dado que, permitió examinar un tema poco estudiado, del cual se tienen muchas dudas y no se ha abordado anteriormente, en este sentido, si bien es cierto que la innovación tecnológica es una temática relativamente estudiada dentro del sector carrocero y específicamente en aquellas empresas consideradas como empresas familiares no es así. Por lo tanto, el estudio tomó como punto de partida aquellos componentes directamente relacionados con las variables, recolectando la mayor cantidad de sucesos que se desarrollan, como requerimiento fundamental para los análisis pertinentes.

En torno a la población de estudio, ésta se estructuró por aquellas empresas clasificadas por el INEC dentro de la categoría Empresas Familiares o Pequeñas Empresas las mismas que cumplían las siguientes características: 
a) capital entre $\$ 100.001$ a $\$ 1.000 .000$ y b) número de empleados entre 10 a 49 personas. Bajo este contexto las empresas que cumplieron estos lineamientos fueron: 1) Mambus, 2) Impedepsa, 3) Alme, 4) Impa y 5) Pillapa. Por lo tanto, al estar constituido la población únicamente por 5 empresas no fue necesario obtener ningún tipo de muestro por lo que se trabajó con la totalidad de la población encontrada.

\section{Resultados y Discusión}

3.1 Diagnóstico de las innovaciones tecnológicas introducidas en la industria carrocera

A nivel de sector se consideran cinco de las principales empresas de la industria carrocera. Cada una de ellas cuenta con importantes ingresos totales anuales, sin embargo, no todas superan los ingresos totales promedio del sector que asciende a \$ 3.716.800 dólares. Al hacer un ranking de las empresas, la primera posición, es decir, la empresa que supera los ingresos totales promedio es ALME con \$ 5.030.000 de dólares anuales.

Tabla 2: Sector ranking por ingresos totales (IT) anuales

\begin{tabular}{|c|c|c|c|c|c|}
\hline Empresa & Tipo de producto & $\begin{array}{c}\text { Venta } \\
\text { Anual (en } \\
\text { unidades) }\end{array}$ & $\begin{array}{l}\text { Precio de } \\
\text { Mercado } \\
\quad \text { (dls.) }\end{array}$ & $\begin{array}{l}\text { Ingreso Total } \\
\text { por Producto } \\
\text { (dls. anuales) }\end{array}$ & $\begin{array}{l}\text { Ingreso Total } \\
\text { por Empresa } \\
\text { (dls. anuales) }\end{array}$ \\
\hline \multirow{3}{*}{ ALME } & Bus Interprovincial & 20 & $\$ 150.000$ & $\$ 3.000 .000$ & \multirow{3}{*}{$\$ 5.030 .000$} \\
\hline & Bus Tipo & 10 & $\$ 125.000$ & $\$ 1.250 .000$ & \\
\hline & Bus Escolar & 6 & $\$ 130.000$ & $\$ 780.000$ & \\
\hline \multirow{3}{*}{ IMPA } & Bus Interprovincial & 10 & $\$ 150.000$ & $\$ 1.500 .000$ & \multirow{3}{*}{$\$ 4.242 .000$} \\
\hline & Bus Tipo & 10 & $\$ 123.000$ & $\$ 1.230 .000$ & \\
\hline & Bus Escolar & 12 & $\$ 126.000$ & $\$ 1.512 .000$ & \\
\hline \multirow{3}{*}{ PILLAPA } & Bus Interprovincial & 14 & $\$ 148.000$ & $\$ 2.072 .000$ & \multirow{3}{*}{$\$ 3.560 .000$} \\
\hline & Bus Tipo & 8 & $\$ 122.000$ & $\$ 976.000$ & \\
\hline & Bus Escolar & 4 & $\$ 128.000$ & $\$ \quad 512.000$ & \\
\hline \multirow{3}{*}{ IMPEDEPSA } & Bus Interprovincial & 10 & $\$ 145.000$ & $\$ 1.450 .000$ & \multirow{3}{*}{$\$ 3.194 .000$} \\
\hline & Bus Tipo & 8 & $\$ 122.000$ & $\$ 976.000$ & \\
\hline & Bus Escolar & 6 & $\$ 128.000$ & $\$ 768.000$ & \\
\hline \multirow{3}{*}{ MANBUS } & Bus Interprovincial & 8 & $\$ 148.000$ & $\$ 1.184 .000$ & \multirow{3}{*}{$\$ 2.558 .000$} \\
\hline & Bus Tipo & 6 & $\$ 121.500$ & $\$ 729.000$ & \\
\hline & Bus Escolar & 5 & $\$ 129.000$ & $\$ \quad 645.000$ & \\
\hline
\end{tabular}

En este sentido, se puede deducir por el momento, que los ingresos de cada una de estas empresas se derivan de la venta de sus productos, las estrategias empleadas para llegar a dichos valores pueden estar asociadas a la venta de un mayor volumen de las unidades más económicas o porque registran una buena venta de las unidades de mayor costo, en ambos casos, ya sea por volumen, por precio o por ambos casos, las empresas incrementan sus ingresos totales al término del año.

De los tres productos en el mercado, en todos los casos, el Bus Interprovincial suele ser el de mayor costo, le sigue el Bus Escolar y finalmente el más económico de los tres constituye el Bus Tipo. En este sentido, de forma general, se observa que ALME representa la empresa que más ingresos anuales registra, además de ser la que más unidades vende en el mercado en sus diferentes productos (bus interprovincial, bus tipo y bus escolar) con un total de 36 unidades vendidas que representa un total de ingresos de $\$ 5.030 .000$. Mientras que MANBUS constituye la empresa dentro del sector que menor producción y comercialización presentan pues de acuerdo a la información recopilada se vendieron un total de 19 unidades en sus distintos productos los mismos que representaron un total de ingresos al año de \$2.558.000 (ver tabla 2).

Por otro lado, en la siguiente tabla se evidencian los Costos Totales (CT) como porcentaje del ingreso total para 
cada uno de los productos que ofrecen las cinco empresas consideradas en el estudio. Asimismo, se presentan las distintas reducciones en costo que cada empresa experimentó entre los años 2013 y 2017. La introducción de nuevas tecnologías en cada empresa tuvo su origen en distintos periodos y esta reducción en costos varía según la empresa.

Tabla 3: Reducción de costos totales por innovación tecnológica por empresa y por tipo de producto

\begin{tabular}{|c|c|c|c|c|}
\hline Empresa & Tipo de producto & $\begin{array}{c}\text { Bus } \\
\text { Interprovincial }\end{array}$ & $\begin{array}{c}\text { Bus } \\
\text { Tipo }\end{array}$ & $\begin{array}{c}\text { Bus } \\
\text { Escolar }\end{array}$ \\
\hline \multirow{3}{*}{ ALME } & $\%$ de costo total respecto del ingreso total en el periodo $t$ & 79 & 80 & 75 \\
\hline & \%Reducción de costos para $(\mathrm{t}+1)$ & 18 & 16 & 19 \\
\hline & \%Reducción de costos para $(\mathrm{t}+2)$ & 21 & 20 & 25 \\
\hline \multirow{3}{*}{ IMPEDEPSA } & $\%$ de costo total respecto del ingreso total en el periodo $t$ & 77 & 82 & 84 \\
\hline & \%Reducción de costos para $2015(\mathrm{t}+1)$ & 13 & 18 & 16 \\
\hline & \%Reducción de costos para $2017(\mathrm{t}+2)$ & 25 & 20 & 19 \\
\hline \multirow{3}{*}{ MANBUS } & $\%$ de costo total respecto del ingreso total en el periodo $\mathrm{t}$ & 74 & 85 & 81 \\
\hline & \%Reducción de costos para $2014(\mathrm{t}+1)$ & 16 & 15 & 19 \\
\hline & \%Reducción de costos para $2017(\mathrm{t}+2)$ & 19 & 18 & 20 \\
\hline \multirow{3}{*}{ IMPA } & $\%$ de costo total respecto del ingreso total en el periodo $t$ & 73 & 84 & 78 \\
\hline & \%Reducción de costos para $2013(\mathrm{t}+1)$ & 27 & 16 & 22 \\
\hline & \%Reducción de costos para $2017(\mathrm{t}+2)$ & 29 & 19 & 23 \\
\hline \multirow{3}{*}{ PILLAPA } & $\%$ de costo total respecto del ingreso total en el periodo $t$ & 79 & 88 & 85 \\
\hline & \%Reducción de costos para $2014(\mathrm{t}+1)$ & 21 & 12 & 15 \\
\hline & \%Reducción de costos para $2017(\mathrm{t}+2)$ & 23 & 15 & 17 \\
\hline
\end{tabular}

En este caso, de acuerdo a la información recopilada, la empresa IMPEDEPSA experimentó una reducción del $25 \%$ de los costos por efecto de la introducción de nuevas tecnologías en el Bus Interprovincial durante el año 2017, mientras que en el año 2015 registró una disminución del $13 \%$. Por su parte, MANBUS, vio una mayor reducción en el producto Bus Escolar, particularmente en el año 2017 con un $20 \%$ menos por efecto de la introducción de nuevas tecnologías y mejoras en este producto. Asimismo, IMPA y PILLAPA presentaron también una reducción en sus costos en el producto Bus Interprovincial con porcentajes del $29 \mathrm{y}$ $23 \%$ respectivamente.

Una vez que se tiene los ingresos totales (IT) y los costos totales (CT), se procede a estimar los beneficios de cada empresa. En este sentido, seguidamente se presenta dicha información de forma ordenada.

Tabla 4: Ingresos totales anuales por innovación tecnológica por empresa y por tipo de producto

\begin{tabular}{|c|c|c|c|c|c|c|c|c|}
\hline \multirow{2}{*}{ EMPRESA } & \multirow{2}{*}{ Tipo de producto } & \multirow{2}{*}{$\begin{array}{l}\text { Ingreso Total (dls. } \\
\text { anuales) }\end{array}$} & Costo Total & \multirow{2}{*}{$\begin{array}{c}\text { Beneficios } \\
(\pi)\end{array}$} & \multirow{2}{*}{$\begin{array}{c}\text { Costo Total } \\
\text { (dls.) }\end{array}$} & \multirow{2}{*}{$\begin{array}{c}\text { Beneficios } \\
(\pi)\end{array}$} & \multirow{2}{*}{$\begin{array}{c}\text { Costo Total } \\
\text { (dls.) }\end{array}$} & \multirow{2}{*}{$\begin{array}{c}\text { Beneficios } \\
(\pi)\end{array}$} \\
\hline & & & (dls.) & & & & & \\
\hline \multirow{4}{*}{ ALME } & Bus Interprovincial & $\$ 3.000 .000$ & $\$ 1.943 .400$ & $\$ 1.056 .600$ & $\$ 1.593 .588$ & $\$ 1.406 .412$ & $\$ 1.258 .935$ & $\$ 1.741 .065$ \\
\hline & Bus Tipo & $\$ 1.250 .000$ & $\$ 840.000$ & $\$ 410.000$ & $\$ 705.600$ & $\$ 544.400$ & $\$ 564.480$ & $\$ 685.520$ \\
\hline & Bus Escolar & $\$ 780.000$ & $\$ \quad 473.850$ & $\$ 306.150$ & $\$ 383.819$ & \$ 396.182 & $\$ 287.864$ & \$ 492.136 \\
\hline & Totales & $\$ 5.030 .000$ & $\$ 3.257 .250$ & $\$ 1.772 .750$ & $\$ 2.683 .007$ & $\$ 2.346 .994$ & $\$ 2.111 .278$ & $\$ 2.918 .722$ \\
\hline \multirow{4}{*}{ IMPEDEPSA } & Bus Interprovincial & $\$ 1.450 .000$ & $\$ 1.116 .500$ & $\$ 333.500$ & $\$ 971.355$ & $\$ 478.645$ & $\$ 728.516$ & \$ 721.484 \\
\hline & Bus Tipo & $\$ 976.000$ & $\$ 800.320$ & $\$ 175.680$ & $\$ 656.262$ & \$ 319.738 & $\$ 525.010$ & $\$ 450.990$ \\
\hline & Bus Escolar & $\$ 768.000$ & $\$ \quad 645.120$ & $\$ 122.880$ & $\$ 541.901$ & \$ 226.099 & $\$ 438.940$ & \$ 329.060 \\
\hline & Totales & $\$ 3.194 .000$ & $\$ 2.561 .940$ & $\$ 632.060$ & $\$ 2.169 .518$ & $\$ 1.024 .482$ & $\$ 1.692 .466$ & $\$ 1.501 .534$ \\
\hline \multirow{4}{*}{ MANBUS } & Bus Interprovincial & $\$ 1.184 .000$ & $\$ 876.160$ & $\$ 307.840$ & \$ 735.974 & \$ 448.026 & $\$ 596.139$ & $\$ 587.861$ \\
\hline & Bus Tipo & $\$ 729.000$ & $\$ \quad 619.650$ & $\$ 109.350$ & $\$ 526.703$ & \$ 202.298 & $\$ 431.896$ & \$ 297.104 \\
\hline & Bus Escolar & $\$ 645.000$ & $\$ 522.450$ & $\$ 122.550$ & $\$ 423.185$ & \$ 221.816 & $\$ 338.548$ & $\$ 306.452$ \\
\hline & Totales & $\$ 2.558 .000$ & $\$ 2.018 .260$ & $\$ 539.740$ & $\$ 1.685 .861$ & $\$ 872.139$ & $\$ 1.366 .583$ & $\$ 1.191 .417$ \\
\hline \multirow{4}{*}{ IMPA } & Bus Interprovincial & $\$ 1.500 .000$ & $\$ 1.095 .000$ & $\$ 405.000$ & $\$ 799.350$ & $\$ 700.650$ & $\$ 567.539$ & $\$ 932.462$ \\
\hline & Bus Tipo & $\$ 1.230 .000$ & $\$ 1.033 .200$ & \$ 196.800 & $\$ 867.888$ & $\$ 362.112$ & $\$ 702.989$ & $\$ 527.011$ \\
\hline & Bus Escolar & $\$ 1.512 .000$ & $\$ 1.179 .360$ & $\$ 332.640$ & $\$ 919.901$ & $\$ 592.099$ & \$ 708.324 & \$ 803.676 \\
\hline & Totales & $\$ 4.242 .000$ & $\$ 3.307 .560$ & $\$ 934.440$ & $\$ 2.587 .139$ & $\$ 1.654 .861$ & $\$ 1.978 .851$ & $\$ 2.263 .149$ \\
\hline \multirow{4}{*}{ PILLAPA } & Bus Interprovincial & $\$ 2.072 .000$ & $\$ 1.636 .880$ & $\$ 435.120$ & $\$ 1.293 .135$ & $\$ 778.865$ & \$995.714 & $\$ 1.076 .286$ \\
\hline & Bus Tipo & $\$ 976.000$ & $\$ \quad 858.880$ & $\$ 117.120$ & $\$ 755.814$ & \$ 220.186 & $\$ 642.442$ & \$ 333.558 \\
\hline & Bus Escolar & $\$ 512.000$ & $\$ \quad 435.200$ & $\$ 76.800$ & $\$ 369.920$ & $\$ 142.080$ & $\$ 307.034$ & \$ 204.966 \\
\hline & Totales & $\$ 3.560 .000$ & $\$ 2.930 .960$ & $\$ 629.040$ & $\$ 2.418 .870$ & $\$ 1.141 .130$ & $\$ 1.945 .190$ & $\$ 1.614 .810$ \\
\hline
\end{tabular}


La disminución de los costos totales por empresa es importante porque guarda una estrecha relación con el incremento en los beneficios económicos de la misma. En este sentido, se supone que los ingresos anuales son constantes, es decir, que la cuota de venta por producto y los precios no presentan una variación significativa, esto permite estimar los beneficios a nivel industria. Para el periodo inicial, el promedio de beneficios anuales para esta industria asciende a \$901.606 dólares. De la misma manera, los beneficios anuales con relación al tiempo $\mathrm{t}+$ 1 presenta una reducción en costos totales lo cual presenta una repercusión positiva en los beneficios de cada empresa y por consiguiente en los beneficios promedios del sector pues refleja un valor general de $\$ 1$ 1.407.921. Finalmente, los beneficios anuales con relación al tiempo $t+2$ de igual forma muestran una reducción paulatina de los costos totales de cada una de las empresas por efecto de las innovaciones tecnológicas aplicadas en el producto final lo cual genera que los beneficios se vayan incrementando de forma positiva y con ello los beneficios del sector también se ven beneficiados dado que para este último escenario el valor obtenido asciende a \$ 1.897.926.

Tabla 5: Comportamiento de las empresas en estudio

\begin{tabular}{lrrrrrr}
\hline \multicolumn{1}{c}{ Empresas } & $\begin{array}{c}\text { Beneficios t } \\
\text { por empresa }\end{array}$ & $\begin{array}{c}\text { Beneficios t } \\
\text { por sector }\end{array}$ & $\begin{array}{c}\text { Beneficios t +1 } \\
\text { por empresas }\end{array}$ & $\begin{array}{c}\text { Beneficios } \\
\mathbf{t}+\mathbf{1} \text { por } \\
\text { sector }\end{array}$ & $\begin{array}{c}\text { Beneficios t + 2 } \\
\text { por empresas }\end{array}$ & $\begin{array}{c}\text { Beneficios } \\
\mathbf{t}+2 \text { por } \\
\text { sector }\end{array}$ \\
\hline ALME & $\$ 1.772 .750$ & & $\$ 2.346 .994$ & & $\$ 2.918 .722$ \\
IMPEDEPSA & $\$ 632.060$ & & $\$ 1.024 .482$ & & $\$ 1.501 .534$ \\
MANBUS & $\$ 539.740$ & $\$ 901.606$ & $\$ 872.139$ & $\$ 1.407 .921$ & $\$ 1.191 .417$ & $\$ 1.897 .926$ \\
IMPA & $\$ 934.440$ & & $\$ 1.654 .861$ & & $\$ 2.263 .149$ \\
PILLAPA & $\$ 629.040$ & & $\$ 1.141 .130$ & & $\$ 1.614 .810$ \\
\hline
\end{tabular}

Conforme mejoran los beneficios por empresa, al momento de promediar, los beneficios promedios anuales del sector también experimentan mejoras significativas. Lo que evidencia que el sector en general presentó un incremento positivo determinando ganancias por efecto de la introducción de innovaciones tecnológicas que permitieron en primera instancia mejorar la calidad de los productos ofertados en el mercado local y además de ello, experimentar una reducción considerable de los costos totales por empresa.

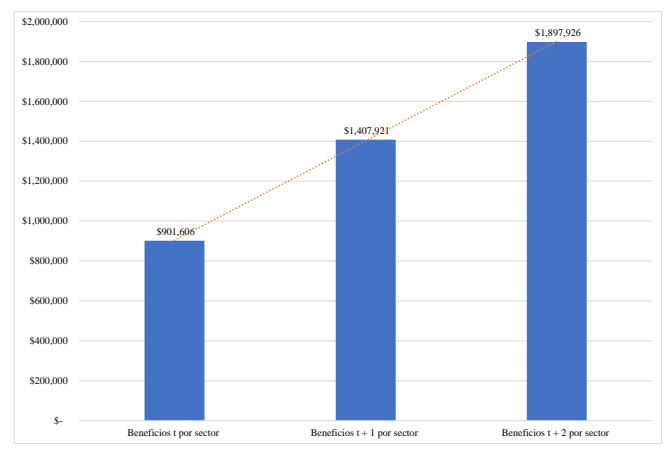

Figura 2: Comportamiento de las empresas en estudio

Lo expuesto en epígrafes anteriores ayuda a corroborar la idea de que las ganancias que cada empresa obtiene abren una posibilidad para que cada una de éstas desarrollen una estrategia de absorción a favor de incrementar la introducción de mejoras en innovaciones tecnológicas. En este sentido, las empresas invierten en relación a las ganancias que obtienen de sus actividades económicas, sin embargo, el presente estudio supone también que dichas empresas no acceden a créditos, sino que van mejorando paulatinamente sus finanzas de acuerdo a las estrategias aplicadas, además de dejar un margen de inversión que pueden destinarlo a innovaciones que les permite mejorar sus productos, continuar con sus tendencia de ventas en el mercado y seguir compitiendo en la industria carrocera.

Las empresas que conforman la industria carrocera que se utilizaron en este estudio reflejan la introducción de nuevas tecnologías en diferentes partes del producto final que se oferta al mercado. En este sentido, cada una de las innovaciones implementadas permitieron reducir los costos de la empresa e incrementar los beneficios económicos de las mismas teniendo la posibilidad de tomar decisiones de inversión para un crecimiento empresarial en el corto, mediano y largo plazo, de esta manera es como se vinculan los beneficios económicos con el crecimiento empresarial (ver figura 3).

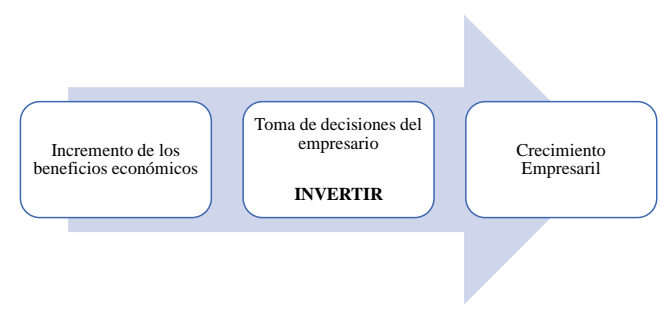

Figura 3: Beneficios económicos y crecimiento empresarial 
Con estos antecedentes se responde a la hipótesis de investigación de que la innovación tecnológica que implementan las empresas del sector carrocero influye en la disminución de sus costos y esto en el incremento de sus beneficios económicos, así, los beneficios económicos que perciben las empresas por esta política tecnología al interior le abren la posibilidad de tomar decisiones, invertir y planear senderos de crecimiento empresarial en el corto, mediano y largo plazo.

\section{Conclusiones}

Las organizaciones que han formalizado sus procesos de innovación, hacen uso de métricas distintas a las convencionales para medir el impacto y los resultados económicos que genera la innovación. En este sentido, estos indicadores se enfocan en la aceptación del mercado de un nuevo producto o servicio, aumentando el volumen de ventas, aumento en la eficiencia en el uso de recursos, rapidez en sacar un producto al mercado, utilización de nuevos canales y apertura hacia nuevos mercados (local, nacional e internacional).

Asimismo, la innovación tecnológica y la competitividad se han considerado como uno de los aspectos más significativas para distintas organizaciones económicas de un país, dado que son elementos de desarrollo en las líneas de ingresos y empleo. Por otro lado, se consideran uno de los principales paradigmas para el desarrollo aplicado a las organizaciones para fortalecer su capacidad productiva $\mathrm{y}$, en consecuencia, mejorar en gran medida su capacidad competitiva.

Finalmente, en cuanto se refiere a la cuantificación de beneficios económicos obtenidos, se pudo determinar que las empresas inmiscuidas en el estudio durante casi dos años destinaron importantes recursos para la adquisición de maquinaria con nuevas mejoras tecnológicas. Sin embargo, las estrategias implementadas por cada una de las empresas fueron diferentes, no obstante, todas éstas fueron vinculadas a la visión que se encuentra direccionada hacia la consecución de la innovación y su mejora en el crecimiento empresarial.

\section{Referencias Bibliográficas}

Bonilla Jurado, D., Oña Sinchiguano, B., y López Núñez, H. (2018). Medición de innovación tecnológica como eje central del crecimiento empresarial familiar del sector carrocero de la Provincia de Tungurahua. Revista Lasallista de Investigación, 15(2), 271-285. doi: 10.22507/rli.v15n2a21

Cadena, J., Pereira, N., y Pérez, Z. (2019). La innovación y su incidencia en el crecimiento y desarrollo de las empresas del sector alimentos y bebidas del Distrito Metropolitano de Quito (Ecuador) durante el 2017. , 40(2017), 17

Carrasco Hernández, A. J., y Sánchez Marín, G. (2014). El capital humano en la empresa familiar: un análisis exploratorio en empresas españolas. Faedpyme International Review, 3(5), 1-10.
Cea, N. (2016). El papel de la innovación y la tecnología en los medios de comunicación: revisión de la literatura académica y propuesta de clasificación. Razón y Palabra(93), 853-867.

Da Silva Tavares, E., y Schneck de Paula Pessoa, M. (2014). Tecnological innovation projects: proposal for an integrative model between project management and knowledge management in a customersupplier perspective. Journal of Information Systems and Technology Management, 11(1), 105-130.

De Massis, A., Frattini, F., y Lichtenthaler, U. (2012). Research on technological innovation in family firms: present debates and future directions. $\mathrm{Fa}$ mily Business Review, 26(1), 10-31. doi: 10.1177/ 0894486512466258

Delfín Pozos, F. L., y Acosta Márquez, M. P. (2016). Importancia y análisis del desarrollo empresarial. Revista científica Pensamiento y Gestión(40), 184202.

Fuentes Lombardo, G., Hernández Ortíz, M., y Vallejo Martos, M. (2008). Razones para crecer en la empresa familiar: un análisis comparativo. Investigaciones Europeas de Dirección y Economía de la Empresa, 14(3), 15-34.

Gómez Luna, E., Fernando Navas, D., Aponte Mayor, G., y Betancourt Buitrago, L. A. (2014). Metodología para la revisión bibliográfica y la gestión de información de temas científicos, a través de su estructuración y sistematización. Dyna, 81(184), 158-163.

Haixing, N. (2018). Study on the role of technological innovation in business administration. Modern Economy(9), 1619-1624. doi: 10.4236/me.2018 .910100

Hernández Sampieri, R., y Mendoza Torres, C. (2018). Metodología de la investigación: Las rutas cuantitativa, cualitativa y mixta (Primera Ed ed.; McGrawHill, Ed.). Ciudad de México: McGrawHill.

Herrera González, R., y Gutiérrez Gutiérrez, J. M. (2015). Conocimiento, Innovación y Desarrollo (Impresión Gráfica del Este, Ed.). San José.

Londoño Rua, J. E. (2015). Tecnología como factor de innovación en empresas colombianas. Revista Venezolana de Gerencia, 20(70), 201-216.

Mantilla, M., Vilcacundo, A., Ruiz, M., y Mayorga, C. (2016). La innovación tecnológica y la competitividad de las Pymes manufactureras del cantón Ambato (Inf. Téc.). Ambato.

Mariz Pérez, R. M., y Calvo Silvosa, A. R. (2015). La estrategia de crecimiento empresarial: Una visión desde la teoría de los costes de transacción. La gestión de la diversidad: XIII Congreso Nacional, 665-670.

Molina Parra, P. A., Botero Botero, S., y Montoya Monsalve, J. N. (2016). Empresas de familia: conceptos y modelos para su análisis. Pensamiento Y Gestión(41), 116-149. 
Perozo, A. J., y Villalobos, R. (2014). Naturaleza de la innovación tecnológica del software como servicio en las organizaciones de desarrollo de software del Municipio Maracaibo. Revista Electrónica de Estudios Telemáticos, 13(2), 36-48.

Quejada Pérez, R. F., y Ávila Gutiérrez, J. N. (2016). Empresas familiares: Conceptos, teorías y estructuras. Revista Escuela de Administración de Negocios(81), 149-158.

Rahman, H., Sanguino Galván, R., y Barroso Martínez, A. (2017). Impact of Family Business on Economic Development: A Study of Spain's Family-owned Supermarkets. Journal of Business and Management Sciences, 5(4), 129-138. doi: 10.12691/jbms-5-4-4

Robayo Acuña, P. V. (2016). La innovación como proceso y su gestión en la organización: una aplicación para el sector gráfico colombiano. Suma de Negocios, 7(16), 125-140. doi: 10.1016/j.sumneg.2016.02.007

Robledo Velásquez, J. (2017). Introducción a la gestión tecnogía y la innovación (Universidad Nacional de Colombia, Ed.). Medellín.

Sandu, P. (2019). A framework of family business professionalization. International Journal of Entrepre- neurship, 23(1), 1-5.

Seclén, J. P. (2016). Gestión de la innovación empresarial: un enfoque multinivel. Revista de Ciencias de la Gestión, 1(1), 16-36.

Vélez Romero, X., y Ortiz Restrepo, S. (2016). Emprendimiento e innovación: Una aproximación teórica. Dominio de las Ciencias, 2(4), 346-369.

Recibido: 5 de enero de 2019

Aceptado: 20 de noviembre de 2019 
